\title{
Stage III Penile Cancer AJCC v7
}

National Cancer Institute

\section{Source}

National Cancer Institute. Stage III Penile Cancer A/CC V7. NCI Thesaurus. Code C89197.

Stage III includes: IIIa (T1-3, N1, M0); IIIb (T1-3, N2, M0). T1: Tumor invades subepithelial connective tissue without lymph vascular invasion and is not poorly differentiated (i.e., grade 3-4) or tumor invades subepithelial connective tissue with lymph vascular invasion or is poorly differentiated. T2: Tumor invades corpus spong iosum or cavernosum. T3: Tumor invades urethra. cN1: Palpable mobile unilateral inguinal lymph node. pN1: Metastasis in a single inguinal lymph node. cN2: Palpable mobile multiple or bilateral inguinal lymph nodes. pN2: Metastasis in multiple or bilateral superficial ing uinal lymph nodes. M0: No distant metastasis. (AJCC 7th ed.) 